\title{
NOTE ON QUASIFIBRATIONS AND MANIFOLDS
}

\author{
RALPH STÖCKER
}

\begin{abstract}
Let $E$ be a closed $C^{\infty}$-manifold which is the total space of a quasifibration over $S^{n}$ with fibre $S^{k}$. Then, in many cases, $E$ has the homotopy type of an orthogonal $S^{k}$-bundle over $S^{n}$. The proof includes a classification theorem for certain quasifibrations which has further applications.
\end{abstract}

1. Introduction. In [3], examples are given of the following phenomena: There exist quasifibrations over $S^{n}$ with fibre $S^{k}$ whose total spaces have the homotopy type of closed $C^{\infty}$-manifolds but not the homotopy type of any $S^{k}$-bundle over $S^{n}$. In fact, for $k=2$ and $4<n \neq \equiv 0 \bmod 4$, the complex $S^{n} \vee S^{2} \cup e^{n+2}$ with the top cell attached by $\left[\iota^{n}, \iota^{2}\right]+\iota^{2} \circ \alpha$ has the desired properties provided that $\alpha \in \pi_{n+1}\left(S^{2}\right), \alpha \neq 0$, and some iterated suspension $E^{N} \alpha=0$. It quasifibers over $S^{n}$ with fibre $S^{2}$ (by Theorem III of [8]), it has the homotopy type of a closed $C^{\infty}$-manifold (by BrowderNovikov theory, for Poincaré duality holds and the Spivak normal spherical fibration is trivial since $\left.E^{N} \alpha=0\right)$ and it does not have the homotopy type of any $S^{2}$-bundle over $S^{n}$ (for this would imply $\alpha=0$; see [3] for details). Thus $S^{k}$-quasifibrations over $S^{n}$ lead to new examples of closed $C^{\infty}$-manifolds, at least if $k=2$ and for some values of $n$. One may ask whether or not the same phenomena occurs for other values of $k$ and $n$. The answer is "no" in the stable range.

THEOREM 1. Let $p: E \rightarrow S^{n}$ be a quasifibration with fibre $\simeq S^{k}$ and $k \geqq n$; then $E$ has the homotopy type of a closed $C^{\infty}$-manifold iff it has the homotopy type of an orthogonal $S^{k}$-bundle over $S^{n}$.

This follows from Theorem 6 below and the fact that if $k \geqq n$ there exists a homotopy section $s: S^{n} \rightarrow E$. We only consider quasifibrations which admit homotopy sections (as the examples above do) and whose total spaces have the homotopy type of a CW-complex. The main step is to classify them, as follows:

Two maps $p: E \rightarrow B$ and $p^{\prime}: E^{\prime} \rightarrow B$ are called equivalent, $p \sim p^{\prime}$, if there exists a homotopy equivalence $h: E \rightarrow E^{\prime}$ such that $p^{\prime} h \simeq p$; observe that

Received by the editors April 10, 1973 and, in revised form, June 1, 1973. AMS (MOS) subject classifications (1970). Primary 55F05, 55F15, 57D10. type.

Key words and phrases. Quasifibration, manifold, orthogonal bundle, fibre homotopy

(C) American Mathematical Society 1974 
this implies fibre homotopy equivalence if $p$ and $p^{\prime}$ are fibrations. Given $\alpha \in \pi_{n+k-1}\left(S^{k}\right), n, k \geqq 2$, let $X_{\alpha}=S^{n} \vee S^{k} \cup e^{n+k}$ with the top cell attached by $\left[\iota^{n}, \iota^{k}\right]+\iota^{k} \circ \alpha$, and let $p_{\alpha}: X_{\alpha} \rightarrow S^{n}$ be a map such that $p_{\alpha} \mid S^{n} \vee S^{k}$ is homotopic to the projection $S^{n} \vee S^{k} \rightarrow S^{n}$. The equivalence class of $p_{\alpha}$ is uniquely determined by $\alpha$. Let $\bar{\alpha}$ be the coset of $\alpha$ modulo the subgroup which consists of all Whitehead products $[\rho, 1] \in \pi_{n+k-1}\left(S^{k}\right)$ with $\rho \in \pi_{n}\left(S^{k}\right)$.

THEOREM 2. (a) The equivalence class of $p_{\alpha}$ contains a quasifibration $p: E \rightarrow S^{n}$ with $p^{-1}(*)=S^{k}$ which admits a homotopy section. (b) Let $\alpha, \beta \in \pi_{n+k-1}\left(S^{k}\right)$; then $p_{\alpha} \sim p_{\beta}$ iff $\bar{\alpha}=\bar{\beta}$ or $\bar{\alpha}=(-(-1) \circ \beta)^{-}$.

Corollary. $\pi_{q}\left(X_{\alpha}\right)=\iota^{n} \circ \pi_{q}\left(S^{n}\right)+\iota^{k} \circ \pi_{q}\left(S^{k}\right)$ if $q>0$, and composition with $\iota^{n}$ and $\iota^{q}$ is injective.

If $n>k+1$, (a) is contained in Theorem III of [8]; the general case is proved in $\$ 2$ below. (b) follows from the definition of equivalence by a standard calculation which is left to the reader (recall $n, k \geqq 2$ ).

Let $p: E \rightarrow S^{n}$ be a quasifibration with fibre $\simeq S^{k}$ which admits a homotopy section. Let $C(p) \subset \pi_{n+k-1}\left(S^{k}\right)$ be the set of all "brace products" of $p$ (compare [4] and $\$ 2$ below).

THeOREm 3. If $\alpha \in C(p)$, then $C(p)$ is the union of the cosets $\bar{\alpha}$ and $(-(-1) \circ \alpha)^{-}$, and $p \sim p_{\alpha}$.

This completes the classification of quasifibrations over $S^{n}$ with fibre $\simeq S^{i}$ which admit homotopy sections up to equivalence: The set of equivalence classes is in 1-1 correspondence with the factor set of $\pi_{n+k-1}\left(S^{k}\right)$ obtained by identifying $\alpha, \beta \in \pi_{n+k-1}\left(S^{k}\right)$ if $\bar{\alpha}=\bar{\beta}$ or $\bar{\alpha}=(-(-1) \circ \beta)^{-}$. The classification by homotopy type is as follows:

THEOREM 4. Let $n \neq k$ or let $n=k$ be even; then $X_{\alpha} \simeq X_{\beta}$ iff $\bar{\alpha}= \pm \bar{\beta}$ or $\bar{\alpha}=( \pm(-1) \circ \beta)^{-}$. Let $n=k$ be odd; then $X_{\alpha} \simeq X_{\beta}$ iff there exists an integer $m$ relatively prime to the order of $\bar{\alpha}$ such that $m \bar{\alpha}=\bar{\beta}$.

The case $n \neq k$ is similar to the proof of (b) of Theorem 2 and is left to the reader; the case $n=k$ is discussed in full detail in [6].

Now let us return to the problem mentioned at the beginning. A quasifibration $p: E \rightarrow S^{n}$ is called smoothable if $E$ has the homotopy type of a closed $C^{\infty}$-manifold $M$; it is called sectionally smoothable if $M$ and a homotopy equivalence $h: E \rightarrow M$ exist such that, for some homotopy section $s: S^{n} \rightarrow E, h s: S^{n} \rightarrow M$ is homotopic to a smooth embedding.

THEOREM 5. A quasifibration over $S^{n}$ with fibre $\simeq S^{k}$ which admits a homotopy section is sectionally smoothable iff it is equivalent to an orthogonal bundle. 
THEOREM 6. Let $n \leqq 2 k-3$; then a quasifibration over $S^{n}$ with fibre $\simeq S^{k}$ which admits a homotopy section is smoothable iff it is equivalent to an orthogonal bundle.

Theorem 6 follows from Theorem 5 and Haefliger's embedding theorem [2]. Theorems 2, 3 and 5 are proved in $\$ 2$. Some further applications are given in $\S 3$.

2. Proofs. We further assume $n, k \geqq 2$. Let $u, q: S^{n-1} \times S^{k} \rightarrow S^{k}$ be maps such that $q$ is the projection $q(x, y)=y$ and $u\left|S^{n-1} \vee S^{k}=q\right| S^{n-1} \vee S^{k}$. Let $E_{u}$ be obtained from $S^{k}$ by attaching $D^{n} \times S^{k}$ by $u$. Let $\chi:\left(D^{n}, S^{n-1}\right) \rightarrow$ $\left(S^{n}, *\right)$ and $\phi: D^{n} \times S^{k} \rightarrow E_{u}$ be identification maps. Let $p_{u}: E_{u} \rightarrow S^{n}$ be $p_{u}\left(S^{k}\right)=*$ and $p_{u} \phi(x, y)=\chi(x)$ if $x \in D^{n}, y \in S^{k}$. By Theorem (1.8) of [5], $p_{u}$ is a quasifibration with fibre $S^{k}$. The cross-section $S^{n} \rightarrow E_{u}$, given by $x \mapsto \phi\left(\chi^{-1}(x), *\right)$, and the inclusion $S^{k} \subset E_{u}$ define an imbedding $S^{n} \vee S^{k} \subset$ $E_{u}$ with complement a $(n+k)$-cell. Thus $E_{u}=S^{n} \vee S^{k} \cup e^{n+k}$ and $p_{u} \mid S^{n} \vee S^{k}$ equals the projection $S^{n} \vee S^{k} \rightarrow S^{n}$. We are going to calculate the attaching map of $e^{n+k}$.

Let $\omega \in \pi_{n}\left(D^{n}, S^{n-1}\right)$ be the class of the identity map, and let

$$
\omega \times 1 \in \pi_{n+k}\left(D^{n} \times S^{k}, W\right) \text { with } W=D^{n} \times * \cup S^{n-1} \times S^{k}
$$

be the cross product of $\omega$ and $1 \in \pi_{k}\left(S^{k}\right)$, as defined in $\S 5$ of [1]. Define a diagram of maps

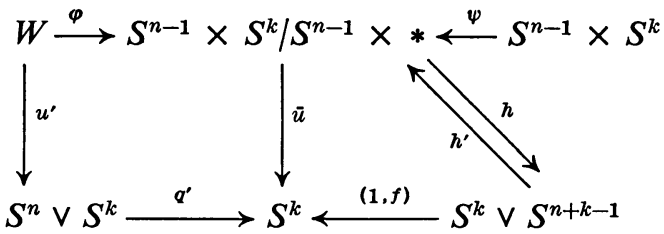

as follows. $\psi$ is the identification map, $\varphi$ its extension to $W$ such that $\varphi\left(D^{n} \times *\right)=* . \bar{u}$ is induced by $u, q^{\prime}$ is the restriction of $q$, and $u^{\prime}$ is the extension of $u$ such that $u^{\prime}(x, *)=\chi(x)$ if $x \in D^{n} . h^{\prime}$ is a map such that $h^{\prime}(y)=$ $\psi(*, y)$ if $y \in S^{k}$, and $h^{\prime} \mid S^{n+k-1}$ represents the element $\varphi_{*} \partial(\omega \times 1)$. By an easy homology argument, $h^{\prime}$ is a homotopy equivalence; define $h$ to be a homotopy inverse such that $h \psi(*, y)=y$ if $y \in S^{k}$. Then $h_{*} \varphi_{*} \partial(\omega \times 1)=$ $\iota^{n+k-1}$, the class of the inclusion $S^{n+k-1} \rightarrow S^{k} \vee S^{n+k-1}$.

The class of the attaching map of the cell $e^{n+k} \subset E_{u}$ is $u_{*}^{\prime} \partial(\omega \times 1)$; from (5.8) of [1] one easily gets

$$
u_{*}^{\prime} \partial(\omega \times 1)=\left[\iota^{n}, \iota^{k}\right]+\iota^{k} \circ q_{*}^{\prime} u_{*}^{\prime} \partial(\omega \times 1) .
$$

Now suppose $u$ is defined by the equation $u=(1, f) h \psi$, where $f: S^{n+k-1} \rightarrow S^{k}$ 
represents a given element $\alpha \in \pi_{n+k-1}\left(S^{k}\right)$. Then

$$
\begin{aligned}
q_{*}^{\prime} u_{*}^{\prime} \partial(\omega \times 1) & =\bar{u}_{*} \varphi_{*} \partial(\omega \times 1)=(1, f)_{*} h_{*} \varphi_{*} \partial(\omega \times 1) \\
& =f_{*}\left(\iota^{n+k-1}\right)=\alpha ;
\end{aligned}
$$

thus the class of the attaching map of $e^{n+k}$ is $\left[\iota^{n}, \iota^{k}\right]+\iota^{k} \circ \alpha$. This shows that the quasifibration $p_{u}$ belongs to the equivalence class of $p_{\alpha}$ of $\S 1$ and proves (a) of Theorem 2 .

We next prove Theorem 3. Let $p: E \rightarrow S^{n}$ be a quasifibration with fibre $\simeq S^{k}$ which admits a homotopy section. If $i: F=p^{-1}(*) \rightarrow E$ denotes the inclusion, there is a split exact sequence $(q>0)$

$$
0 \longrightarrow \pi_{q}(F) \stackrel{i_{*}}{\longrightarrow} \pi_{q}(E) \stackrel{p_{*}}{\longrightarrow} \pi_{q}\left(S^{n}\right) \longrightarrow 0 .
$$

Define $C(p) \subset \pi_{n+k-1}\left(S^{k}\right)$ as follows: Choose a homotopy section $s: S^{n} \rightarrow E$ and a homotopy equivalence $b: S^{k} \rightarrow F$. Denote by $\sigma \in \pi_{n}(E)$ and $\beta \in \pi_{k}(F)$ the homotopy class of $s$ and $b$, respectively. Since the Whitehead product $\left[\sigma, i_{*}(\beta)\right] \in \pi_{n+k-1}(E)$ is mapped to 0 under $p_{*}$, the element

$$
c(p ; s, b)=-b_{*}^{-1} i_{*}^{-1}\left[\sigma, i_{*}(\beta)\right] \in \pi_{n+k-1}\left(S^{k}\right)
$$

is well defined. Let $C(p)$ be the set of all $c(p ; s, b)$, with $s$ and $b$ varying. If $b^{\prime}: S^{k} \rightarrow F$ is a homotopy equivalence, $b^{\prime} \simeq b$ or $b^{\prime} \simeq b d$, where $d: S^{k} \rightarrow S^{k}$ represents -1 . In the first case $c\left(p ; s, b^{\prime}\right)=c(p ; s, b)$, in the second

$$
c\left(p ; s, b^{\prime}\right)=-d_{*}^{-1} b_{*}^{-1} i_{*}^{-1}\left[\sigma,-i_{*}(\beta)\right]=-(-1) \circ c(p ; s, b) .
$$

If $s^{\prime}: S^{n} \rightarrow E$ is a homotopy section too, its homotopy class has the form $\sigma+i_{*} b_{*}(\rho)$ for some $\rho \in \pi_{n}\left(S^{k}\right)$, by the exact sequence above. Conversely, given $\rho$, each map representing $\sigma+i_{*} b_{*}(\rho)$ is a homotopy section. Now clearly the calculation

$$
c\left(p ; s^{\prime}, b\right)=-b_{*}^{-1} i_{*}^{-1}\left[\sigma+i_{*} b_{*}(\rho), i_{*}(\beta)\right]=c(p ; s, b)-[\rho, 1]
$$

proves the first part of Theorem 3 .

Let $\alpha=c(p ; s, b) \in C(p)$, and let $f: S^{n} \vee S^{k} \rightarrow E$ be the map which is $s$ on $S^{n}$ and $b$ on $S^{k}$. Since

$$
f_{*}\left(\left[\iota^{n}, \iota^{k}\right]+\iota^{k} \circ \alpha\right)=\left[\sigma, i_{*}(\beta)\right]+i_{*} b_{*}(\alpha)=0,
$$

$f$ extends to a map $X_{\alpha} \rightarrow E$ (recall the definition of $X_{\alpha}$ in $\S 1$ ). By the exact sequence above and the corollary of Theorem 2 , any extension $X_{\alpha} \rightarrow E$ of $f$ is a weak homotopy equivalence, hence a homotopy equivalence since we assume $E$ to have the homotopy type of a CW-complex. By standard obstruction theory, an extension $g: X_{\alpha} \rightarrow E$ of $f$ exists such that $p g \simeq p_{\alpha}$. Hence $p \sim p_{\alpha}$, in the sense of $\S 1$, which completes the proof of Theorem 3 .

Lemma 1. Let $p: E \rightarrow S^{n}$ be an orthogonal $S^{k}$-bundle over $S^{n}$ which admits a cross-section, and suppose $\delta \in \pi_{n-1}(S O(k))$ maps to a characteristic 
element of $p$ in $\pi_{n-1}(S O(k+1))$. Then $J(\delta) \in C(p)$, with $J: \pi_{n-1}(S O(k)) \rightarrow$ $\pi_{n+k-1}\left(S^{k}\right)$ the Hopf-Whitehead homomorphism.

Proof. Let $f: S^{n-1} \rightarrow S O(k+1)$ with $f(*)=1$ represent the image of $\delta$, and let $u: S^{n-1} \times S^{k} \rightarrow S^{k}$ be the adjoint map $u(x, y)=f(x)(y)$. Then $u=q$ on $S^{n-1} \vee S^{k}$, and therefore $p_{u}: E_{u} \rightarrow S^{n}$ is defined and is an orthogonal $S^{k}$-bundle over $S^{n}$ isomorphic to $p: E \rightarrow S^{n}$ (compare $\$ 3$ of [6]). As is well known, $\bar{f}=u \psi^{-1} h^{\prime} \mid S^{n+k-1}$ represents $J(\delta)$. Then $(1, \bar{f}) h \psi \simeq u \operatorname{rel} S^{n-1} \times *$ and this yields $p \sim p_{u} \sim p_{J(\delta)}$, by calculating $u_{*}^{\prime} \partial(\omega \times 1)$ as in the proof of Theorem 2. Now the lemma follows from Theorems 2 and 3.

Remark. Since $((-1) \circ J(\delta))^{-}=(-J(\delta))^{-}$, by $(2.2)$ of [6], the coset $C(p)=(J(\delta))-$ is (up to an inclusion) just the $\lambda(p)$ of [6].

Proof OF Theorem 5. Let $\alpha \in \pi_{n+k-1}\left(S^{k}\right), n, k \geqq 2$, and suppose there exists a closed $C^{\infty}$-manifold $M$ and a homotopy equivalence $h: X_{x} \rightarrow$ $M$ such that the element $h_{*}\left(\iota^{n}\right) \in \pi_{n}(M)$ is represented by a smoothly imbedded sphere $S^{n} \subset M$.

Lemma 2. Then there exists an orthogonal $S^{k}$-bundle $p: E \rightarrow S^{n}$ such that $p \sim p_{\alpha}$.

This of course implies Theorem 5 .

Proof of Lemma 2. Let $v$ be the normal disc bundle of $S^{n}$ in $M$ with characteristic element $\delta \in \pi_{n-1}(S O(k))$. Its total space may be identified with the closure $\bar{U}$ of an open tubular neighborhood $U$ of $S^{n}$ in $M$. Let $q: M \rightarrow M /(M-U)$ be the projection onto the Thom space $M /(M-U)$ of $v$. By Lemma 1 of [7] there exists a homotopy equivalence $s: M /(M-U) \rightarrow S^{k} \cup e^{n+k}$, where the cell $e^{n+k}$ is attached by $J(\delta)$. Thus we get a map $f=s q h: X_{\alpha} \rightarrow S^{k} \cup e^{n+k}$ which we may assume to be cellular. Let $g: S^{n} \vee S^{k} \rightarrow S^{k}$ be the map defined by $f$. Since $f_{*}: H_{n+k}\left(X_{\alpha}\right) \rightarrow$ $H_{n+k}\left(S^{k} \cup e^{n+k}\right)$ is an isomorphism

$$
g_{*}\left(\left[\iota^{n}, \iota^{k}\right]+\iota^{k} \circ \alpha\right)=\varepsilon J(\delta) \quad \text { with } \varepsilon= \pm 1 .
$$

Furthermore, as will be shown below, $g_{*}\left(\iota^{k}\right)=\varepsilon^{\prime}= \pm 1$. Hence

$$
g_{*}\left(\left[\iota^{n}, \iota^{k}\right]+\iota^{k} \circ \alpha\right)=\left[\varepsilon^{\prime} g_{*}\left(\iota^{n}\right), 1\right]+\left(\varepsilon^{\prime}\right) \circ \alpha,
$$

which together with the first equation implies $\left(J\left(\varepsilon \varepsilon^{\prime} \delta\right)\right)^{-}=\left(\varepsilon^{\prime}\left(\varepsilon^{\prime}\right) \circ \alpha\right)^{-}$. Thus if $p: E \rightarrow S^{n}$ is the orthogonal $S^{k}$-bundle over $S^{n}$ whose characteristic element is the image of $\varepsilon \varepsilon^{\prime} \delta$, we get $p \sim p_{\alpha}$ by Lemma 1 and Theorems 2 and 3.

Let $\mathscr{H}$ be the natural homomorphism from homotopy to homology groups. To prove $g_{*}\left(l^{k}\right)= \pm 1$ it is sufficient to show that $f_{*} \mathscr{H}\left(\iota^{k}\right)$ generates $H_{k}(Y)$. This, since $s$ is a homotopy equivalence and by excision, is equivalent to the statement that $j_{*} h_{*} \mathscr{H}\left(c^{k}\right)$ generates $H_{k}(M, M-U)$ with $j:(M, \varnothing) \rightarrow(M, M-U)$ the inclusion. Let $w \in H_{n+k}\left(X_{\alpha}\right)$ be a generator, 
and let $v \in H^{n}\left(X_{\alpha}\right)$ be dual to $\mathscr{H}\left(\iota^{n}\right) \in H_{n}\left(X_{\alpha}\right)$; thus the Kronecker product $\left(\mathscr{H}\left(\iota^{n}\right), v\right)=1$. Then, as follows from the cell decomposition of $X_{\alpha}$, the cap product $v \cap w= \pm \mathscr{H}\left(l^{k}\right)$. Hence $y \cap z= \pm h_{*} \mathscr{H}\left(l^{k}\right)$, if $z \in$ $H_{n+k}(M)$ is a generator and if $y \in H^{n}(M)$ is dual to $h_{*} \mathscr{H}\left(\iota^{n}\right) \in H_{n}(M)$. Therefore we have to prove that $j_{*}(y \cap z)$ generates $H_{k}(M, M-U)$. By excision, this is equivalent to the following: If $z^{\prime}$ generates $H_{n+k}(\bar{U}, \partial U)$ and if $i: \bar{U} \rightarrow M$ is the inclusion, then $i^{*}(y) \cap z^{\prime}$ generates $H_{k}(\bar{U}, \partial U)$. This is true by Lefschetz duality, since $i^{*}(y)$ generates $H^{n}(\bar{U})$.

3. Further applications. First, from the cell decomposition of $X_{\alpha}$ and the Corollary of Theorem 2, we get the following examples, generalizing Theorem (2.10) of [3]:

THEOREM 7. Let $\alpha \in \pi_{n+k-1}\left(S^{k}\right), n, k \geqq 2$. If $n=k$ is even, suppose the Hopf invariant $H(\alpha)=0$. Then $X_{\alpha}$ and $S^{n} \times S^{k}$ have isomorphic homotopy groups and integral cohomology rings, but are of different homotopy type if $\bar{\alpha} \neq 0$.

The next theorems concern the connection between homotopy type and fibre homotopy type. Instead of Theorems 2 and 3 one may use [9] (and Theorem 4) to prove them; but this is too difficult a way to get the results below, since it includes a deep theorem of G. W. Whitehead (Theorem (3.2) of [10], as corrected in [11]). Observe that our proofs use only elementary homotopy constructions.

The difficult point in Theorems 2 and 3 is the calculation of the automorphism $\alpha \mapsto(-1) \circ \alpha$ of $\pi_{n+k-1}\left(S^{k}\right)$. In the special case $k=2, n \geqq 2$, this automorphism is the identity, which implies

THEOREM 8. Fibrations which admit cross-sections, with fibre a 2-sphere and with base any sphere, are fibre homotopically equivalent, if their total spaces are of the same homotopy type.

This fails to be true if cross-sections do not exist.

The fibrations of Theorem 8 are interesting for the following reason. Let $p: E \rightarrow S^{n}$ be a $S^{2}$-fibration with cross-section which is not trivial (in the sense of fibre homotopy type). Then $p$ is not locally trivial if $n \geqq 3$. For otherwise its structure group could be reduced from Top $\left(S^{2}\right)$ to $O(2)$ contradicting $\pi_{n-1}(O(2))=0$ if $n \geqq 3$ (compare the proof of Theorem (2.6) of [3]). The lowest dimensional example of such a fibration, whose fibre homotopy type does not contain any locally trivial fibration, occurs if $n=3$ : It is $p_{\alpha}$ with $\alpha$ the generator of $\pi_{4}\left(S^{2}\right)=Z_{2}$. Its total space $X_{\alpha}$ is a 1-connected 5-dimensional Poincaré duality space which does not have the homotopy type of a closed $C^{\infty}$-manifold (compare [3]; this does not follow from our results). 
Let $p: E \rightarrow S^{n}$ be a fibration with fibre $\simeq S^{k}$ which admits a crosssection. Let $p_{m}$ be induced from $p$ by a map $S^{n} \rightarrow S^{n}$ of degree $m$. If $\alpha \in C(p)$, then $m \alpha \in C\left(p_{m}\right)$, as easily follows from the definition of $C(p)$. Hence we get, from Theorems 2, 3, 4,

THEOREM 9. Let $p: E \rightarrow S^{n}, p^{\prime}: E^{\prime} \rightarrow S^{n}$ be fibrations which admit crosssections with fibre $\simeq S^{k}$. Then, if $E \simeq E^{\prime}, p^{\prime}$ is induced from $p$ (up to fibre homotopy equivalence) by some map $S^{n} \rightarrow S^{n}$.

COROLlaRIES. (a) If E has the homotopy type of an orthogonal bundle, then $p$ has the fibre homotopy type of an orthogonal bundle.

(b) If $E \simeq S^{n} \times S^{k}$, then $p$ is trivial (in the sense of fibre homotopy type).

\section{REFERENCES}

1. A. L. Blakers and W. S. Massey, Products in homotopy theory, Ann. of Math. (2) 58 (1953), 295-324. MR 15, 731.

2. A. Haefliger, Plongements différentiables de variétés dans variétés, Comment. Math. Helv. 36 (1961), 47-82. MR 26 \#3069.

3. P. J. Hilton and J. Roitberg, Note on quasifibrations and fibre bundles, Illinois $\mathrm{J}$. Math. 15 (1971), 1-8. MR 42 \#8494.

4. I. M. James, On the decomposability of fibre spaces, The Steenrod Algebra and its Applications (Proc. Conf. to Celebrate N. E. Steenrod's Sixtieth Birthday, Battelle Memorial Inst., Columbus, Ohio, 1970), Lecture Notes in Math., vol 168, Springer, Berlin, 1970, pp. 125-134. MR 43 \#4038.

5. - The transgression and Hopf invariants of a fibration, Proc. London Math. Soc. (3) 11 (1961), 588-600. MR 24 \#A3650.

6. I. M. James and J. H. C. Whitehead, The homotopy theory of sphere bundles over spheres. II, Proc. London Math. Soc. (3) 4 (1955), 148-166. MR 16, 948.

7. J. W. Milnor, On the Whitehead homomorphism J, Bull. Amer. Math. Soc. 64 (1958), 79-82. MR 21 \#1594.

8. S. Sasao, On homotopy groups of certain complexes, J. Fac. Sci. Univ. Tokyo Sect. I 8 (1960), 605-630. MR 23 \#A1376.

9. J. Stasheff, A classification theorem for fibre spaces, Topology 2 (1963), 239-246. MR 27 \#4235.

10. G. W. Whitehead, On products in homotopy groups, Ann. of Math. (2) 47 (1946), 460-475. MR 8, 50.

11. J. H. C. Whitehead, On certain theorems of G. W. Whitehead, Ann. of Math. (2) 58 (1953), 418-428. MR 15, 642.

Mathematik Institut, Ruhr-Universitat, D-463 Bochum, West Germany 